\title{
Phenotypic Stability and Genome-Wide Association Study of Late Blight Resistance in Potato Genotypes Adapted to the Tropical Highlands
}

\author{
Hannele Lindqvist-Kreuze, Manuel Gastelo, Willmer Perez, Gregory A. Forbes, \\ David de Koeyer, and Merideth Bonierbale
}

All authors: International Potato Center, Apartado 1558, Lima 12, Peru; and fifth author: Potato Research Centre, Agriculture and Agri-Food Canada, 850 Lincoln Rd., Fredericton, New Brunswick, Canada.

Accepted for publication 1 January 2014.

\section{ABSTRACT}

Lindqvist-Kreuze, H., Gastelo, M., Perez, W., Forbes, G. A., de Koeyer, D., and Bonierbale, M. 2014. Phenotypic stability and genome-wide association study of late blight resistance in potato genotypes adapted to the tropical highlands. Phytopathology 104:624-633.

Potato genotypes from a breeding population adapted to tropical highlands were analyzed for the stability of late blight resistance and also for marker-phenotype association. We harmonized the historical evaluation data, consisting of observations spanning 6 years from two field sites utilizing a resistance scale constructed by comparing the area under the disease progress curve (AUDPC) values of 172 genotypes with that of susceptible control 'Yungay'. In total, 70 potato genotypes had a coefficient of variability $<0.5$ and were considered stable across the environments tested. A principal component analysis demonstrated that the ensemble of experiments formed two distinct groups that reflect the stability of genotype resistance to late blight. Phytophthora infestans isolates present in the experimental fields belonged to the EC-1 clonal lineage and showed variation in virulence beyond the concept of the avirulence determined by the conventionally used R1-R11 differential set. A single-nucleotide polymorphism (SNP) marker on chromosome 9 was associated with late blight resistance and linked to instability. Genotypes with either AACC or AAAC combinations for this SNP were highly resistant only in some environments, while the genotypes with the AAAA combination had more moderate levels of resistance but were stable across environments.
Late blight caused by the oomycete Phytophthora infestans is one of the main constraints for potato production worldwide, causing yearly losses estimated at over $€ 10$ billion (16). Host resistance would be the most effective way to control the disease but efforts in this area have been hampered by the rapid evolution of $P$. infestans, leading to new pathogen strains which overcome host resistance (9). Conventional theory suggests that the most extreme type of resistance is based on the gene-for-gene concept and is effective only against races of the pathogen that carry and express an avirulence (Avr) allele that is compatible with a major resistance $(R)$ gene in the host. Although $R$ gene resistance provides an extreme resistance phenotype, it is vulnerable to changes in the pathogen population (8).

The late blight resistance phenotype in potato has commonly been classified into two categories, vertical and horizontal, where vertical resistance refers to $R$ gene resistance, as described above (9). As noted, vertical ( $R$ gene) resistance is generally expressed as complete, with little or no disease symptoms, although vertical resistance may also have a partial phenotype. Nonetheless, vertical resistance is generally measured as present or absent. Horizontal resistance is, in theory, effective against all pathogen races and is expressed as a rate-reducing resistance phenotype. Horizontal resistance is generally assessed by a synoptic measure of disease progress over time, either by measuring the rate of disease

Corresponding author: H. Lindqvist-Kreuze

E-mail address: h.lindqvist-kreuze@ @ cgiar.org

* The $\boldsymbol{e}$-Xtra logo stands for "electronic extra" and indicates that the online version contains two supplementary figures and four supplementary tables.

http://dx.doi.org/10.1094/PHYTO-10-13-0270-R

(C) 2014 The American Phytopathological Society increase or the area under the disease progress curve (AUDPC). Recently, an interval scale was proposed for converting synoptic resistance measures to more easily interpretable values between 0 and 9 (41). This scale has an interval structure (34), is anchored to a control cultivar to account for environmental effects, and is thought to provide a robust comparison of genotypes' susceptibility to $P$. infestans among years and locations.

The theory for the genetic control of vertical resistance, defined by major $R$ genes that recognize the corresponding Avr alleles of the pathogen, is broadly accepted, and a number of genes such as those from different Solanum spp. have been identified (37). On the other hand, different opinions exist regarding the genetic control of horizontal resistance (33). Horizontal resistance is assumed to be quantitatively inherited and is frequently referred to as quantitative resistance. The two resistance phenotypes may be very difficult to distinguish in the field (31), although some researchers have proposed that careful examination of the time of disease initiation and the rate of disease development can give insight into the type of resistance present (2).

In the 1980s, the International Potato Center (CIP) adopted a potato breeding strategy designed to avoid the incorporation of $R$ genes into a population under improvement for horizontal resistance. The rationale for the exclusion of $R$ genes from the population was to avoid the extreme resistance phenotype that would mask the expression of horizontal resistance in the recurrent selection program. The resulting population was known as "B3" and was derived from a wide range of late blight resistance sources (21). Late blight resistance evaluation was regularly carried out in highland tropical environments of Peru with high endemic disease pressure from the "new" (32) population of $P$. infestans (26).

Environmental conditions can influence the expression of quantitative resistance (36) and, therefore, genotype-environment 
(G-E) interaction is an important factor to consider when measuring potato late blight resistance $(10,17,24,39)$. Furthermore, instability of $R$-gene-based resistance is very sensitive to pathogen population dynamics. A $\mathrm{G}-\mathrm{E}$ analysis that combines several genotypes evaluated in various environments enables the stability analysis of genotypes' performance and may help to select genotypes suitable for given environments. Recent immigrations and the rise of novel $P$. infestans clonal populations as, for example, KE-1 in Africa (28), and super blight races such as 13_A2 in China (23), highlight the importance of studying stability of resistance in relation to pathogen dynamics.

The objectives of the study were to (i) characterize the stability of the late blight resistance phenotype of elite tetraploid potato genotypes adapted to the tropical highlands by analyzing historical field data from experiments conducted over 6 years in two tropical highland locations where late blight is endemic, (ii) relate this stability or lack thereof to changes in the P. infestans populations present in the field, and (iii) identify marker-phenotype associations utilizing the Infinium 8303 Potato Array platform.

\section{MATERIALS AND METHODS}

Resistance data. Historical data from replicated field trials of the late blight resistance evaluations of the tetraploid B3 population between years 2001 and 2006 were retrieved from the CIP corporate database. The two evaluation sites were always the same: Oxapampa $\left(12^{\circ} 34^{\prime} 05^{\prime \prime}\right.$ south, $75^{\circ} 24^{\prime} 23^{\prime \prime}$ west $)$ and Comas $\left(11^{\circ} 46^{\prime} 0^{\prime \prime}\right.$ south, $75^{\circ} 5^{\prime} 0^{\prime \prime}$ west). In the field, each genotype was represented by an experimental unit consisting of 10 plants planted in a plot of $3.00 \mathrm{~m}^{2}$. The order of genotypes was randomized and there were two biological replicates. In total, 10 to 12 genotypes were planted together and surrounded by susceptible infector plants to assure uniform inoculum throughout the field. Disease severity had been recorded visually as percentage of foliage affected at 6- to 8-day intervals until the susceptible control had reached $100 \%$ infection. All trials included the same control genotypes: 'Yungay' (moderately susceptible), 'Kory' (resistant), and 'Amarilis' (moderately resistant). The raw data were visualized using a box plot to detect outliers. Genotypes with no replication, a high standard deviation (stdev/mean $>0.33$ ) for the AUDPC among replicates, or that were absent from more than two experiments were not considered for the analysis. The total dataset contained 195 potato genotypes evaluated in each of the following nine field experiments: Oxapampa (Oxa)2001, 2002, 2005, and 2006 and Comas 2001, 2002, 2004, 2005, and 2006.

Resistance was evaluated using the susceptibility scale (41), which we here call the scale AUDPC (sAUDPC) and which was based on the AUDPC. The AUDPC values were transformed to the susceptibility scale using the moderately susceptible control Yungay as a reference genotype, which had been assigned a scale value of 6 based on its performance relative to highly susceptible genotypes (unpublished data). The formula for transformation was $s A U D P C x=(A U D P C x / A U D P C$ Yungay $) \times 6$, where $s A U D P C x$ is the scale value of the genotype in question, $A U D P C x$ is the AUDPC of the genotype in question, and AUDPCYungay is the AUDPC of Yungay. The stability of resistance was estimated by calculating the coefficient of variation $(\mathrm{CV})$ of the sAUDPC for each genotype across the nine field experiments based on the variance among experiment-level means and the overall mean.

Pairwise correlations for sAUDPC values between experiments were plotted with the R Gclus package (http://cran.r-project.org/ web/packages/gclus/gclus.pdf). Principal component analysis (PCA) was run on sAUDPC values for all experiments with FactoMineR (18). Only the genotypes that had no missing data (in total, $n=172$ ) were used for the correlation analysis and PCA.

$\boldsymbol{P}$. infestans isolates. Isolates $(n=230)$ were collected between 1999 and 2006 from both evaluation sites. Leaflets containing a single lesion were incubated at 15 to $18^{\circ} \mathrm{C}$ in a sealed water-agar petri dish to promote sporulation. Isolates were recovered from the leaves using a filter system, as described previously (26). Isolates were characterized for mating-type, restriction fragment length polymorphism with probe RG-57, and mitochondrial DNA haplotype, as described previously $(11,12,26)$.

$\boldsymbol{R}$ gene differential sets in the field. A host differential set containing plants with 11 previously identified $R$ genes from the Mexican species Solanum demissum was planted in each trial. The plant material was obtained from the Research Institute for Plant Protection, Wageningen, The Netherlands: R1 (CEBECO43154-5; CIP 800986), R2 (CEBECO-44158-4; CIP 800987), R3 (CEBECO-4642-1; CIP 800988), R4 (CEBECO-4431-5; CIP 800989), R5 (Black 3053-18; CIP 800990), R6 (Black XD2-21; CIP 800991), R7 (Black 2182ef(7); CIP 800992), R8 (Black 2424a(5); CIP 800993), R9 (Black 2573; CIP 800994), R10 (Black 3618ad(1); CIP 800995), and R11 (Black 5008ab(6); CIP 800996). Tubers produced from in vitro plants of each differential were planted in the same number of replications as each genotype in the experiments and were evaluated in the same way as the test genotypes.

Genotypic data. In 2012, 103 of the potato genotypes were growing in the field and, thus, were available for DNA extraction. Genotyping was outsourced as a service to Michigan State University. Infinium Potato Array (7) that contains 8,303 SNPs distributed genome wide was used and the raw data of the 103 potato genotypes were processed together with genotypic data from the homozygous $S$. tuberosum Group Phureja DM1-3 516R44 (DM) clone previously subject to whole-genome sequencing (40). Six diploid genotypes, and two additional tetraploid genotypes not part of the B3 population, were included as controls for assessing SNP quality. Two diploid clones were genotyped three times and three tetraploid clones were genotyped twice. The normalized allele signal ratio (normalized $\mathrm{X}$ divided by the sum of normalized $\mathrm{X}$ and normalized $\mathrm{Y}$ ) was calculated using Illumina GenomeStudio software (Illumina, Inc., San Diego, $\mathrm{CA}$ ) and exported in csv format. A custom R script was used to call and filter the genotypes. Each SNP was run through the CodomMarker function of the fitTetra R package (41). Each sample was categorized into one of five possible tetraploid genotypes (AAAA, AAAB, AABB, ABBB, or BBBB). SNPs were then passed through filters testing for the following conditions: DM homozygosity (AA or BB), expected genotypes for diploids $(\mathrm{AA}, \mathrm{AB}, \mathrm{BB})$, each replicated sample had the same genotype call, localization of the SNP to only one genome location (7), and $<5 \%$ of the genotype calls missing. In all, 3,192 SNPs passing these filters were included in the subsequent analysis. Because $\mathrm{DM}$ is a double monoploid, the assumption was that the SNP should be homozygous in this genotype and, therefore, markers that were heterozygous in DM were excluded from the analysis. Furthermore, the analysis included only bi-allelic SNP.

Population structure, association and linkage disequilibrium. The SNP marker order on the physical map of potato was obtained by BLASTn analysis using the 101-bp-long fragment flanking the SNP (http://solcap.msu.edu/potato_infinium.shtml) as query against the $S$. tuberosum Group Phureja DM1-3 516R44 (CIP801092) version 3 DM, version 2.1.10 pseudomolecules (http://potatogenomics.plantbiology.msu.edu/index.html). The SNP tagged all 12 chromosomes with 163 to 299 markers per chromosome. In total, 243 markers were unmapped.

The potato population structure was characterized by STRUCTURE software (27) on the basis of a subset of 120 SNP markers. Ten markers distributed along each of the 12 chromosomes were selected for this analysis and the data were handled as tetraploid. Three repetitive runs were made using a burn-in time of 1,000,000 and 20,000 Markov Chain Monte Carlo repetitions. The allele frequencies were assumed to be correlated among populations and the structure was determined by assuming 1 to 10 subpopulations. The most probable number of populations was 
determined by plotting the natural logarithm (ln) likelihood against the number of subpopulations by Structure Harvester (6) and the examination of the Q matrices of each run. A kinship matrix was calculated using SPAGeDi (15) and, for the association analysis, the negative values were replaced by zero.

Marker phenotype associations were determined with TASSEL software (3). The markers were coded as diploids even though the initial marker identification was done at the tetraploid level. Therefore, all three possible heterozygous genotypes (AAAB, $\mathrm{AABB}$, and $\mathrm{ABBB}$ ) for bi-allelic SNP were coded the same (AB). The entire marker-genotyped set of 103 potato genotypes was analyzed for sAUDPC in each experiment, although the dataset contained some missing data. The mixed linear model took into account the population structure $(\mathrm{Q}$ matrix) and kinship $(\mathrm{K}$ matrix) and was run with the optimum level of compression. The variance components were estimated after each marker. The significance of the test was set to 0.05 and Bonferroni correction was applied to correct for multiple testing and against the identification of false positives. Minor allele frequency ( $>5 \%$ ) was taken into account as a criterion for calling significant markers. The marker association was tested for PCA coordinates of the first dimension (Dim 1) and the second dimension (Dim 2) as well as the CV but using only those 88 potato genotypes that had no missing phenotypic data.

The linkage disequilibrium (LD) coefficient $\left(r^{2}\right)$ was plotted against the physical marker order and the statistical parameters given by TASSEL were used to determine whether markers were in $\mathrm{LD}$ with respect to one another. The significance level was set to $P<0.001$ and corrected for the number of markers analyzed for each chromosome. The physical distances between the markers varied on each chromosome because some adjacent markers may be found within the same gene while others may be millions of base pairs apart.

The Hardy-Weinberg equilibrium was calculated by SNP-HWE for R (http://www.sph.umich.edu/csg/abecasis/Exact/r_instruct.html).

\section{RESULTS}

Pathogen population. All isolates collected from potato fields from Comas $(n=91)$ and Oxapampa $(n=139)$ belonged to the clonal lineage EC-1 and were mating type A1. In all trials, the differential plants with R1, R2, R3, R4, R6, R7, R10, and R11 became infected with late blight. The differential R5 was infected in four experiments (Comas2004, Comas2005, Oxa2001, and Oxa2005), R8 in eight experiments (all but Oxa2005), and R9 in two experiments (Comas2001 and Comas2002).

Resistance. Most of the breeding materials included in this study had AUDPC values lower than the susceptible control genotype Yungay (Supplementary Table 1). However, pairwise correlation analysis of the sAUDPC values indicated that the genotypes' relative levels of resistance varied considerably among experiments (Supplementary Figure 1; Supplementary Table 2), raising concerns about the stability of the resistance. Based on a bi-plot of sAUDPC CV and mean values (Fig. 1), we identified a suitable cut-off level for stability at $\mathrm{CV}=0.5$. Thus, 70 genotypes were considered stable and 102 unstable. Stability of genotypes was also evident by plotting sAUDPC values for locations (Fig. 2 ). Stable genotypes generally had similar values across environments, except in Comas2005. In contrast, unstable genotypes had more variability in mean sAUDPC values among locations, generally having higher values (more susceptible) in four locations (Comas2004, Oxa2001, Oxa2002, and Oxa2005) and also, to a lesser extent, in Oxa2006. Yungay, that was used to calculate the sAUDPC and the relative CV, had a low CV (0.14) of the absolute AUDPC values (Supplementary Table 2).

Plotting locations on the first two dimensions of the PCA, which explained $\approx 80 \%$ of the information in the dataset, produced a pattern that was generally consistent with the classification of locations described above (compare Figs. 2 and 3). The PCA separated locations into two groups: group 1 consisted of the environments Oxa2001, Oxa2002, and Oxa2005 and group 2 contained the remaining locations (Fig. 3). Thus, based on common association with Dim 2 of the PCA, it appears that resistance in the unstable genotypes broke down in group 1 environments (Fig. 4). This loss of resistance of unstable genotypes in group 1 locations did not appear to be related to the pathogen virulence pattern determined by the differential set. A common factor for the group 1 environments was that the R9 differential remained uninfected in all of them; however, this is also the case for four out of six of the group 2 environments (Fig. 3).

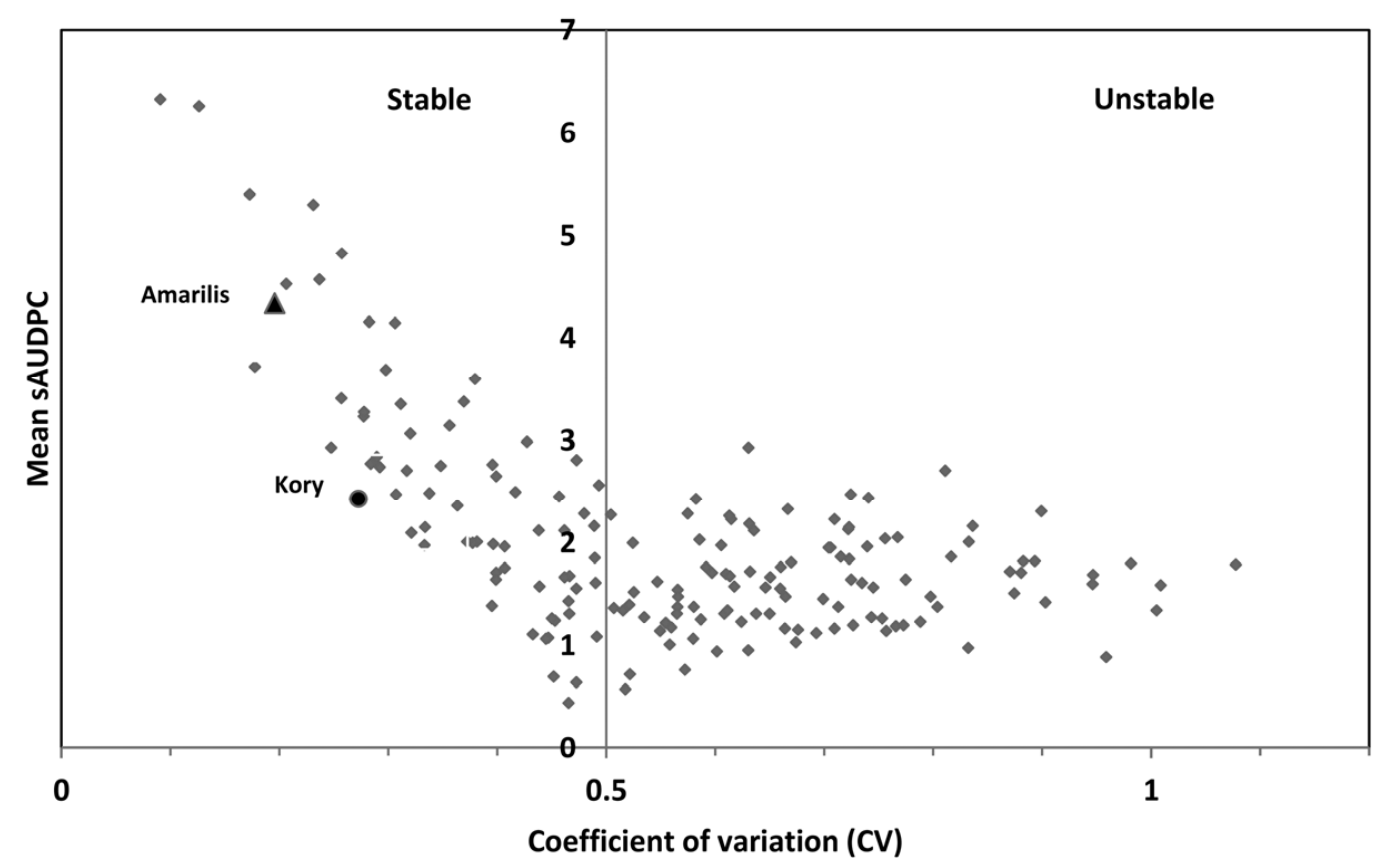

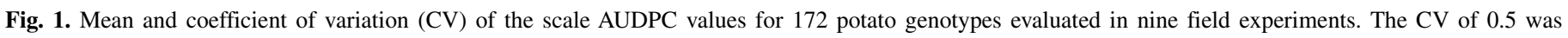
considered a cut-off value between stable and unstable genotypes. 
Host population structure. Based on 120 SNP markers and 103 potato genotypes, the most probable number of subpopulations in our dataset is three (Supplementary Figure 2), although there are genotypes with fairly mixed backgrounds (Fig. 5). Population structure was linked to the marker c2_56418 (see below) because the group of potato genotypes that are heterozygous for this marker (in total, 56 genotypes) belong mostly to the subpopulations 3 (28 genotypes) and 2 (21 genotypes) (Fig. 6).

Marker-trait association. Two markers on chromosome 9 and one marker on chromosome 7 had significant association with the

Stable
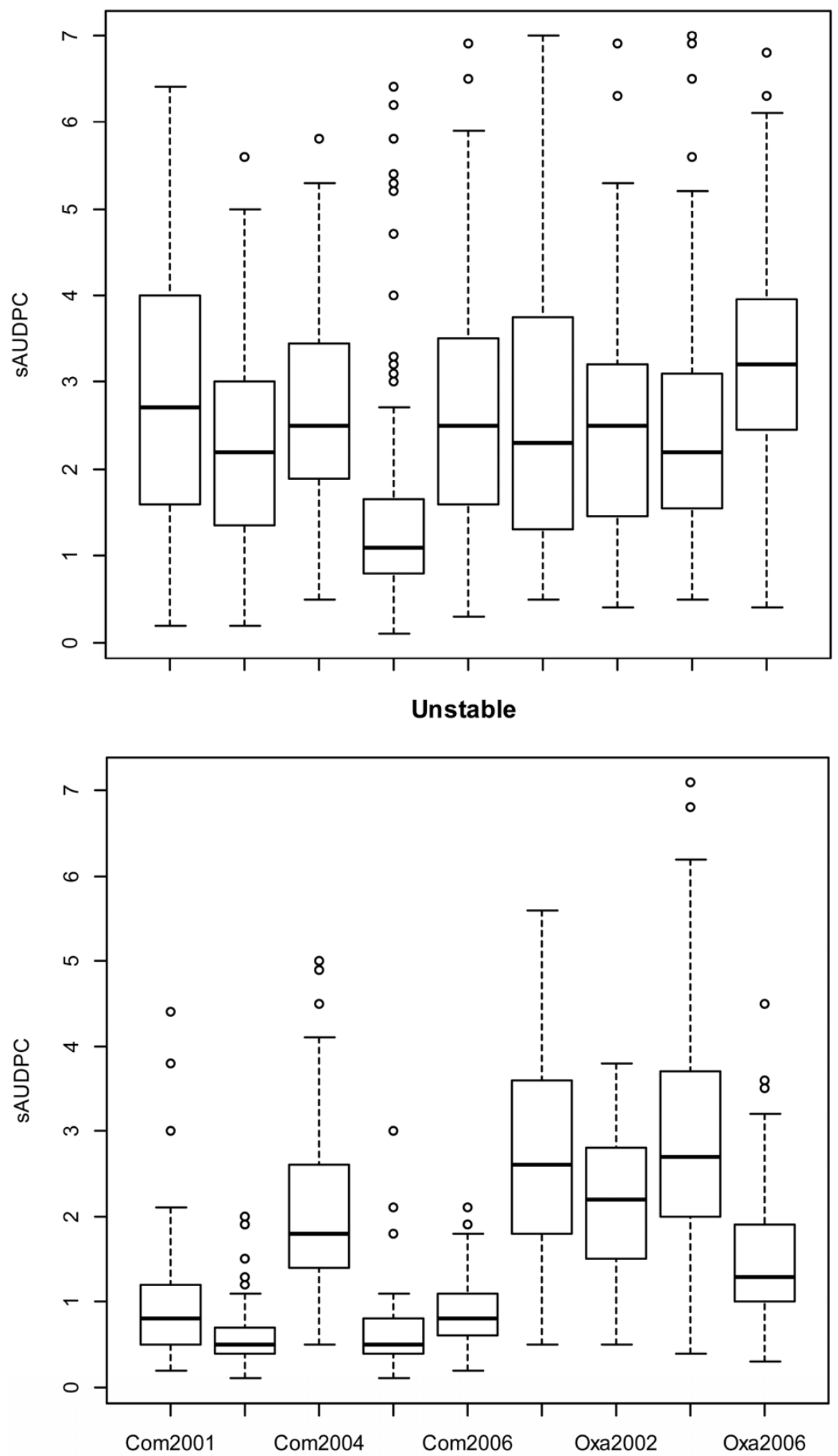

Fig. 2. Pattern of the scale AUDPC values of the stable and unstable genotypes in each experiment. The experiments from left to right are Comas2001, Comas2002, Comas2004, Comas2005, Comas2006, Oxa2001, Oxa2002, Oxa2005, and Oxa2006. The number of race-specific (RS) resistance individuals was included in the graph showing the unstable individuals. 
late blight resistance phenotype (Table 1). Marker c2_56418 was significantly associated with sAUDPC in Comas2006, Oxa2006, and Comas2002 and with Dim 2 of the PCA. This SNP is present in homozygous (AAAA) and heterozygous (AAAC or AACC) states. Potato genotypes with the heterozygous SNP genotype for c2_56418 are more resistant in the group 2 environments
(Oxa2006, Comas2001, Comas2002, Comas2004, Comas2005, and Comas2006) (Fig. 3) and more susceptible in the group 1 environments (Fig. 7). Heterozygous genotypes also have much higher frequency of the founders 575049 (CEW-69-1) and BULK MEX in their pedigree (Table 2; Supplementary Tables 3 and 4). Furthermore, in Oxa2006, where marker c2_56418 explained

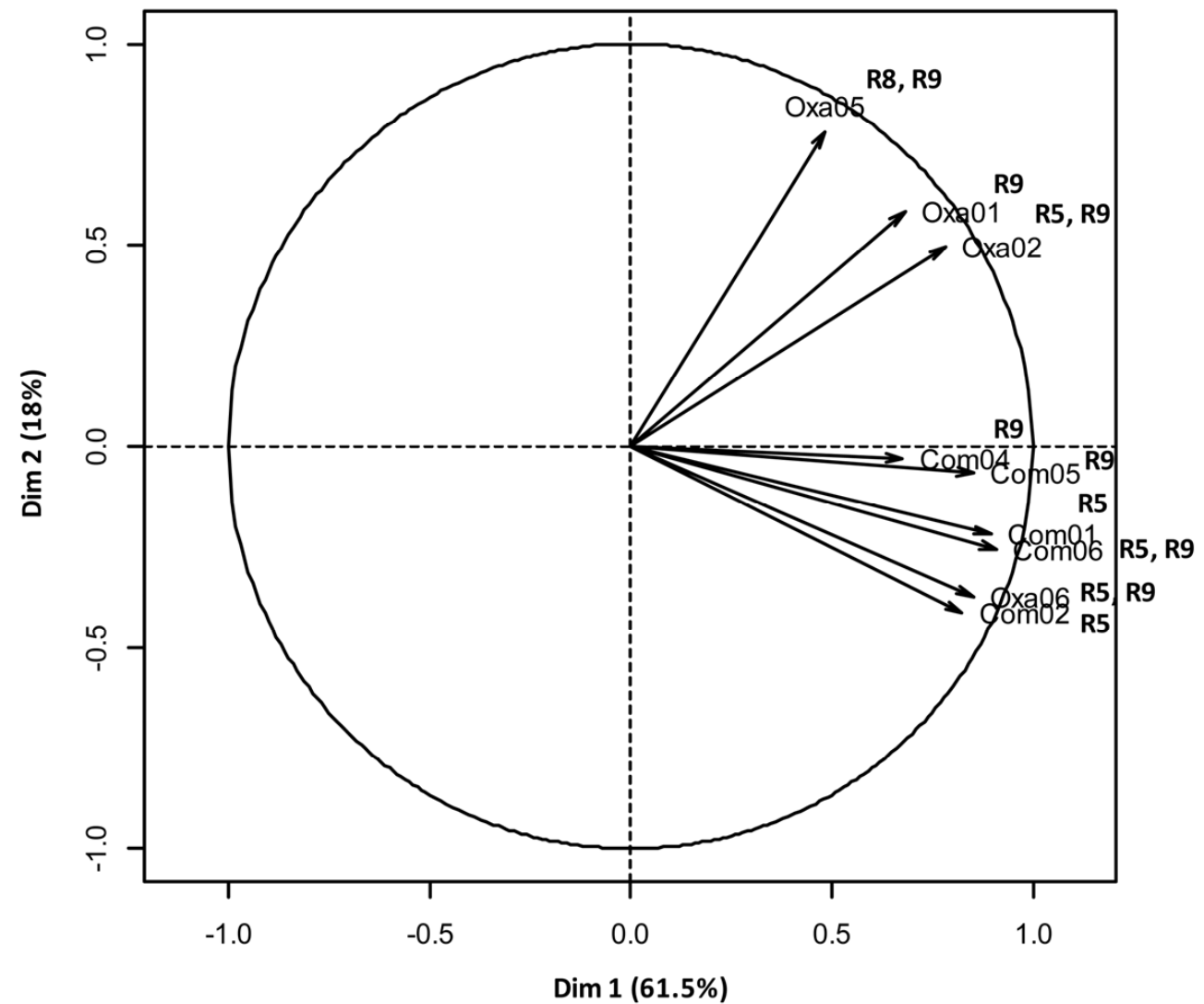

Fig. 3. Bi-plot of the first two dimensions of a principal component analysis showing the relationships among environments where resistance to potato late blight based on the scale AUDPC was evaluated in 172 potato genotypes. Differential set plants R1 to R11 that did not become infected during each experiment are shown after the name of the experiments. Experiments Oxa01, Oxa02, and Oxa05 formed group 1 and the rest of the experiments formed group 2.

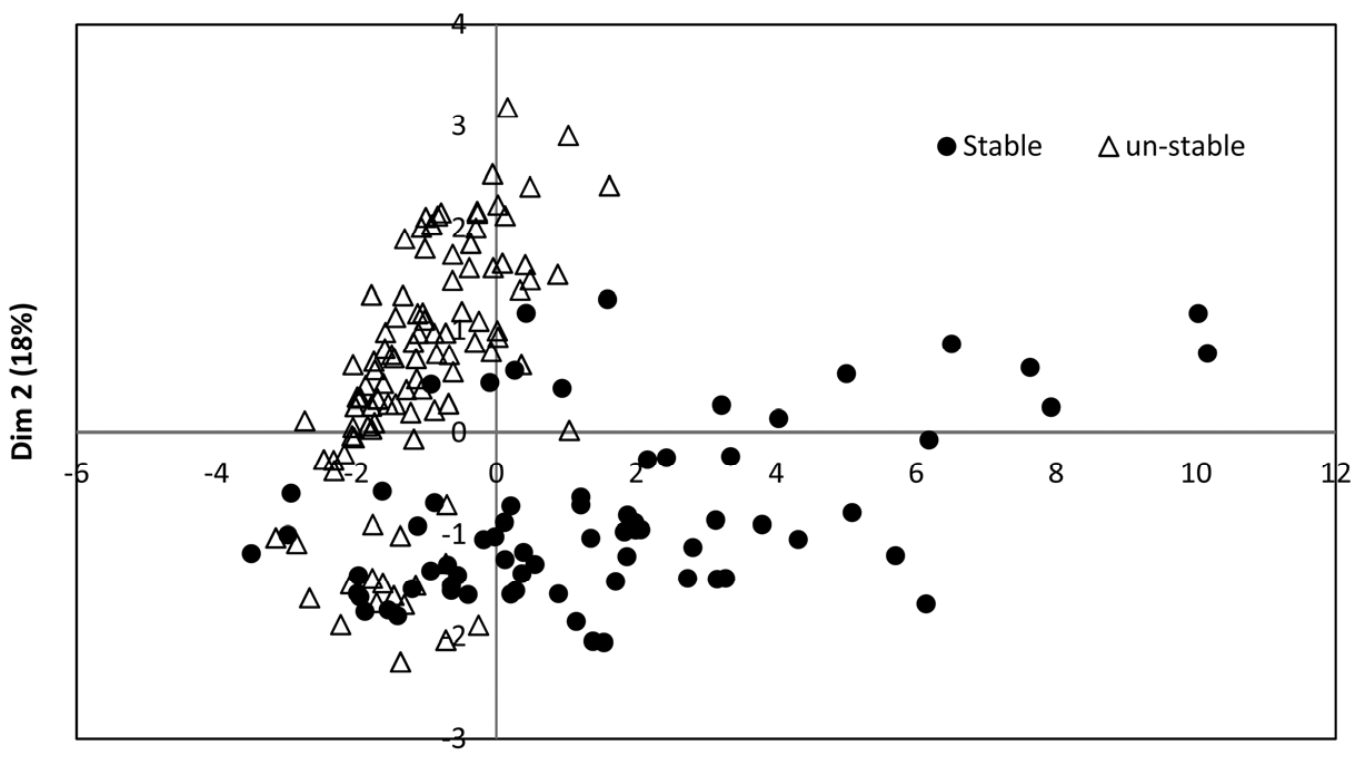

$\operatorname{Dim} 1$ (61.5\%)

Fig. 4. Bi-plot of first two dimensions (Dim 1 and Dim 2) of a principal component analysis based on scale AUDPC values of 172 potato genotypes evaluated for late blight resistance in nine environments. Genotypes are identified based on stability; genotypes with an among-experiment coefficient of variation for sAUDPC of $<0.5$ were considered stable. 
$21 \%$ of the variation, there are 31 potato genotypes with sAUDPC value $\leq 1$, and 29 of those are heterozygous for the c2_56418 SNP. Of those, all but three have the founders 575049 (CEW-69-1) or BULK MEX in their pedigree. Most potato genotypes that are heterozygous for this marker locus are unstable for the sAUDPC value (Fig. 8).

The marker c2_56418 is located toward the end of the long arm of chromosome 9 but has not been mapped to any gene in the reference potato genome assembly. The physical distance of c2_56418 from its neighboring markers is $96 \mathrm{~kb}$ to the south (c1_10509) and $538 \mathrm{~kb}$ to the north (c1_14205), and neither of these two markers is in LD with c2_56418 (Fig. 9). It is not surprising that the latter one is not in LD with the associated marker because of the large physical distance between them, which also contains a gap of unknown size in the potato genome sequence. As an example of the degree of LD nearby, three markers (c2_27699, c2_27705, and c1_8549) toward the end of the chromosome are in absolute LD and the physical distance between the first and last of these markers is $110 \mathrm{~kb}$ (Fig. 9). Also, marker c2_27763 in Sucrose Synthase gene PGSC0003DMG400006672 that is associated with sAUDPC in one of the experiments (Comas2006) has been genetically mapped to chromosome 9 (14) but is unmapped in the genome assembly; therefore, we cannot compare its physical location in relation to the marker c2_56418.

Marker c1_10011 is associated with the sAUDPC in Comas2002 and with the Dim 1 of PCA (Table 1). The SNP is in the gene PGSC0003DMG400000689, Glucan endo-1,3- $\beta$-glucosidase, in chromosome 7 . However, this marker needs to be tested with a higher number of genotypes because there are too few individuals in one of the allele groups, making it difficult to draw meaningful conclusions regarding the SNP allele effect (Table 1).

\section{DISCUSSION}

Increasing the level of late blight resistance in potato has been one of the main priorities of the CIP for decades. Early on, resistance provided by the $S$. demissum $R$ genes was found to be ephemeral and, therefore, a strategy was formulated in the $1980 \mathrm{~s}$ to avoid the incorporation of the major-effect $R$ genes into potato populations bred for horizontal resistance (putatively resistant against all pathogen genotypes). In addition to the short-lived resistance, $R$ genes tended to mask horizontal resistance and, therefore, made improvement for horizontal resistance impossible. To eliminate $R$ genes, potential parents were test-crossed with a potato variety compatible with a putatively "simple" race (also known as race 0 and unable to infect a potato plant with any of the then-known $R$ genes from $S$. demissum); if the resulting progeny had a hypersensitive reaction or no sporulation when inoculated with the simple race, the parent was not used for further crossing (21). Knowledge of both host and pathogen genetics has evolved since the B3 population was designed and

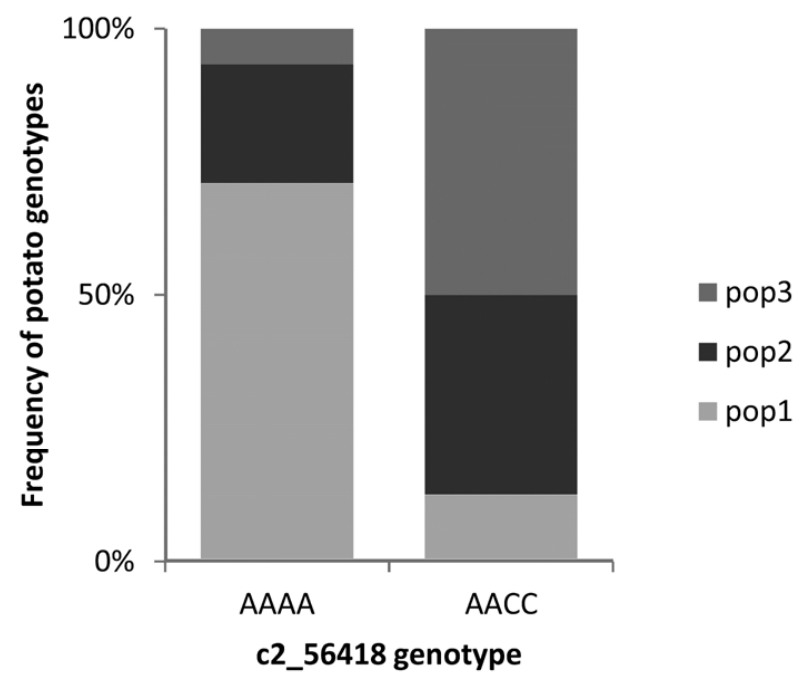

Fig. 6. Potato genotypes divided according to the snp_c2_56418 genotype and population structure assuming three subpopulations.

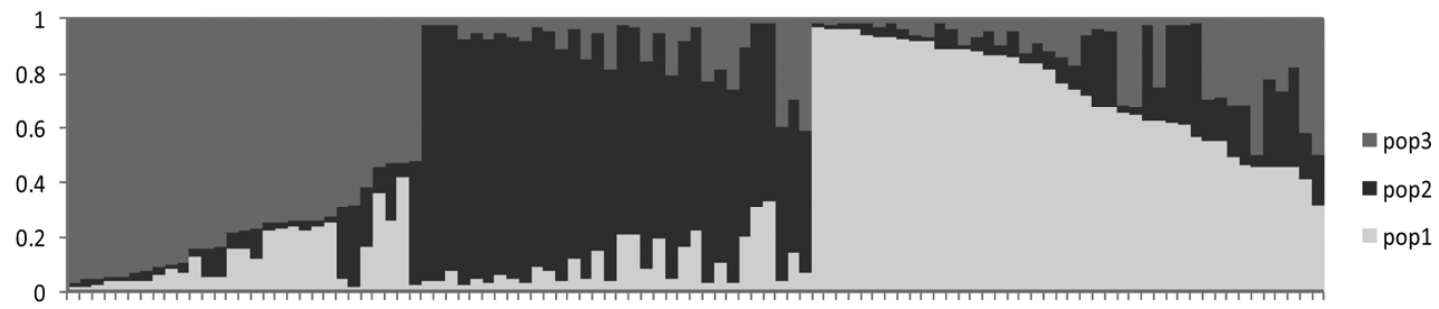

Fig. 5. Structure plot of the assignment probabilities of the 103 potato genotypes. Each genotype is represented by a bar and the highest Q group membership defines subpopulation (pop1, pop2, and pop3) assignment. The plot is based on 120 single-nucleotide polymorphisms and the highest probability run for $\mathrm{K}=3$.

TABLE 1. Significant $(P<0.05)$ single-nucleotide polymorphism $(\mathrm{SNP})$ associations for late blight resistance measured as scale AUDPC (sAUDPC) and the first two principal component dimensions (Dim 1 and Dim 2) in the nine field experiments

\begin{tabular}{|c|c|c|c|c|c|c|c|c|}
\hline \multirow[b]{2}{*}{$\mathrm{Chr}^{\mathrm{a}}$} & \multirow[b]{2}{*}{ SNP_ID ${ }^{\mathrm{b}}$} & \multicolumn{2}{|c|}{ Genotypic class } & \multirow[b]{2}{*}{ HWE deviation ${ }^{\mathrm{e}}$} & \multirow[b]{2}{*}{ Significant $\mathrm{f}^{\mathrm{f}}$} & \multicolumn{2}{|c|}{ sAUDPC mean } & \multirow[b]{2}{*}{$\mathrm{PC}^{\mathrm{g}}$} \\
\hline & & $\mathrm{AA}^{\mathrm{c}}$ & $\mathrm{AB}^{\mathrm{d}}$ & & & $\mathrm{AA}$ & $\mathrm{AB}$ & \\
\hline \multirow[t]{2}{*}{7} & c1_10011 & 15 & 87 & $*$ & Comas2002 & 2.3 & 0.9 & Dim 1 \\
\hline & & & & & Comas2006 & 2.0 & 0.8 & \\
\hline & & & & & Comas2002 & 1.8 & 0.7 & \\
\hline & c2_277663 & 27 & 74 & $*$ & Comas2006 & 2.2 & 1.0 & \\
\hline
\end{tabular}

a Potato chromosome.

b SNP marker name.

c Number of potato genotypes with the homozygous allele composition.

d Number of potato genotypes with the heterozygous allele composition.

e Asterisk indicates significant deviation and ns indicates no significant deviation from Hardy-Weinberg equilibrium $(\mathrm{HWE})$ at $P<0.001$.

${ }^{\mathrm{f}}$ Field experiment with significant marker-trait association.

g Principal component.

${ }^{\mathrm{h}}$ Chromosome location from Hackett et al. (14). 
additional $R$ genes from other species than $S$. demissum have been identified (30). It now appears evident that this strategy to exclude the 11 known $S$. demissum $R$ genes only eliminated some of the $R$ genes in the B3 population.

The nine field experiments in our study formed two groups but the grouping does not strictly follow the geographical location of the evaluation sites. Although sites are situated in the Andes, they are geographically distant and are also in different agroecologies. In Comas, potato is commonly grown by local farmers whereas, in Oxapampa, potato is only grown in home gardens, which could potentially have an impact on the source, quantity, and genetic diversity of the inoculum. However, in both environments, late blight epidemics are severe and $P$. infestans strains found from cultivated potato in both locations were of the EC-1 clonal lineage, which is consistent with previous reports from the Andean countries (26).

TABLE 2. Frequency of potato clones that contain the two founders, 575049 and BULK MEX, in their pedigree grouped by snp_c2_56418 genotype

\begin{tabular}{lccc}
\hline $\begin{array}{l}\text { snp_c2_56418 } \\
\text { genotype }\end{array}$ & $n$ & $\begin{array}{c}575049(\text { CEW-69-1) } \\
(\%)\end{array}$ & $\begin{array}{c}\text { BULK MEX } \\
(\%)\end{array}$ \\
\hline AACC/AAAC & 56 & 63 & 59 \\
AAAA & 45 & 33 & 27 \\
\hline
\end{tabular}

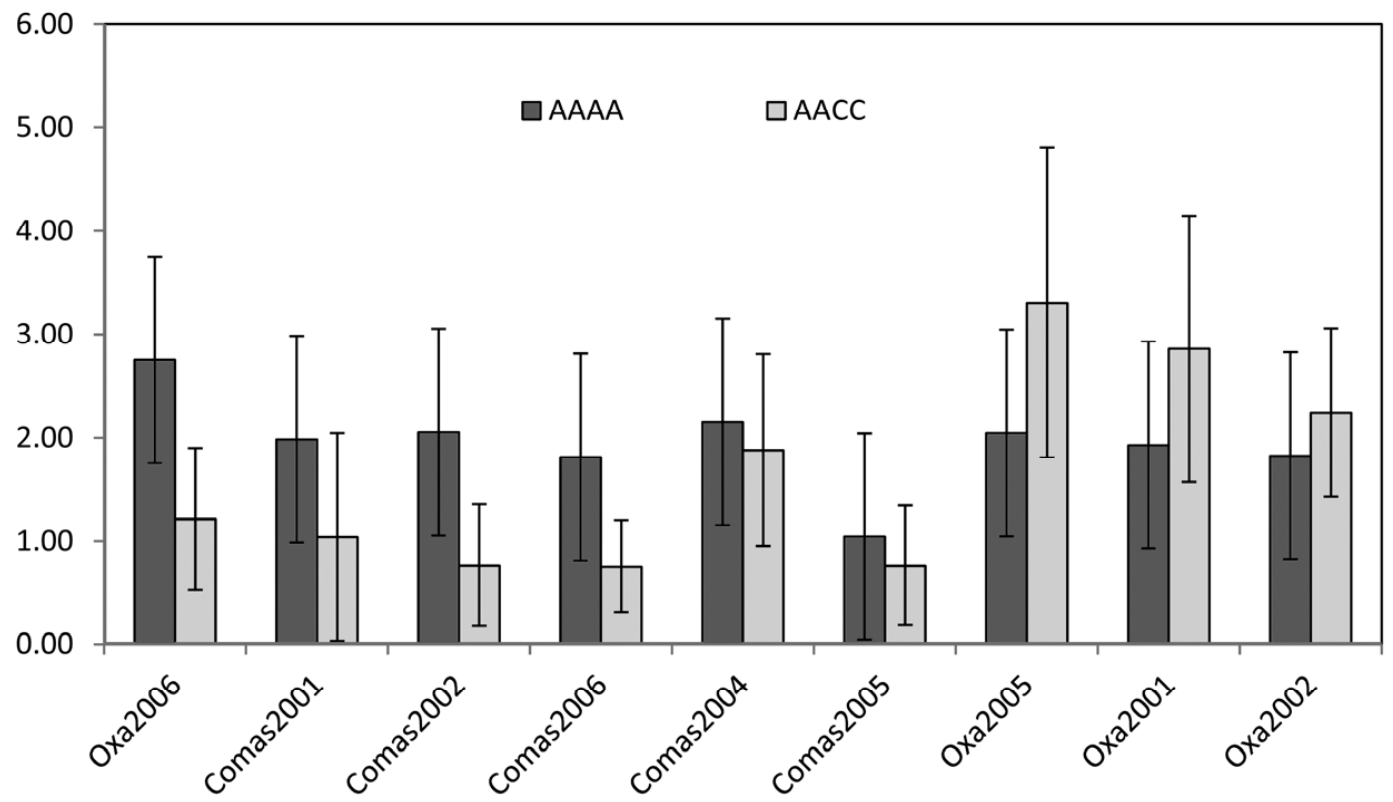

Fig. 7. Single-nucleotide polymorphism (SNP) c2 56418 allele effect on resistance measured as scale AUDPC values (y-axis) showing the mean sAUDPC values of the potato genotypes with SNP genotype AAAA $(n=45)$ versus AACC and AAAC $(n=56)$ in each environment. The first six experiments form group 2 and the last three form group 1 in principal component analysis (Fig. 3).

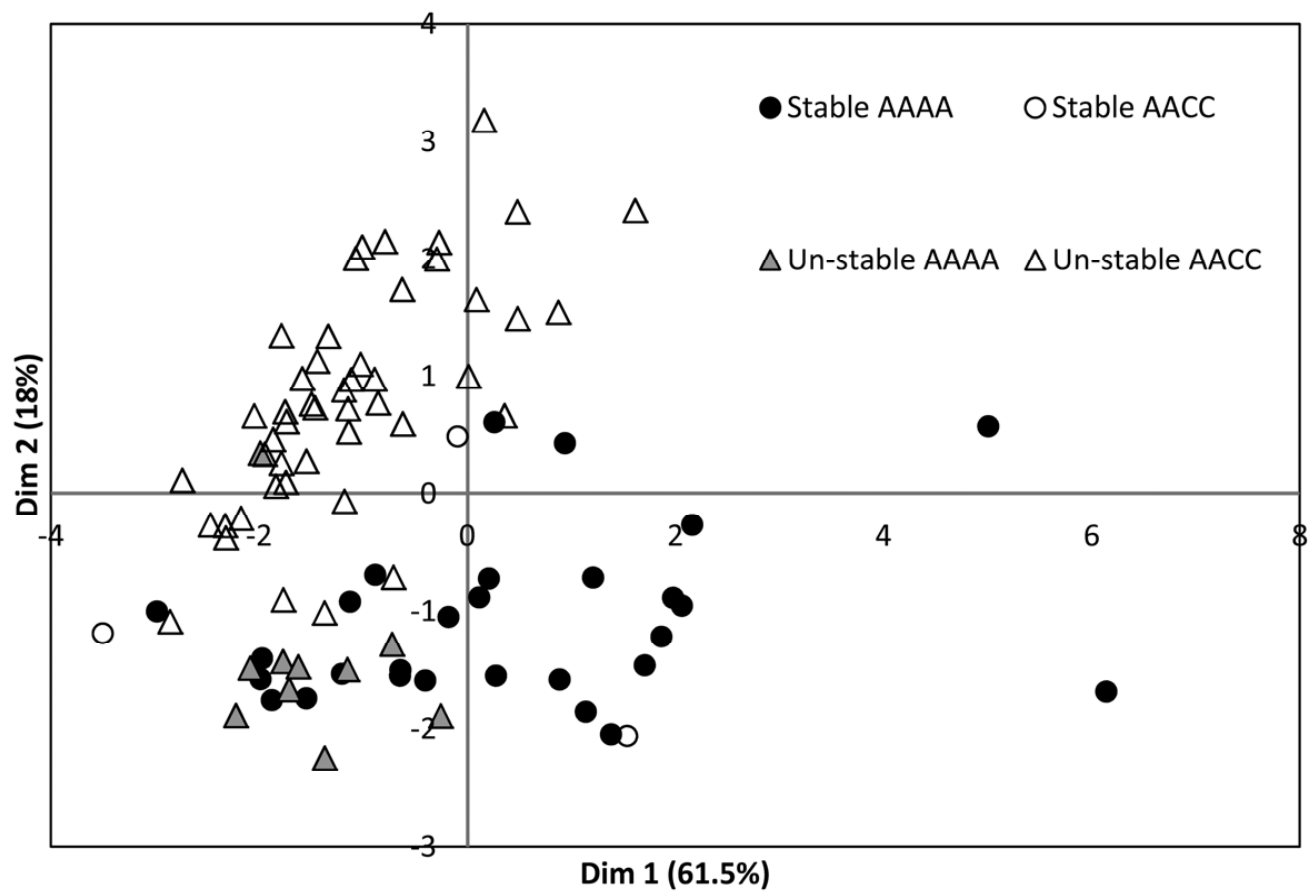

Fig. 8. Bi-plot of first two dimensions (Dim 1 and Dim 2) of a principal component analysis based on scale AUDPC values of only the potato genotypes that were single-nucleotide polymorphism (SNP) genotyped and had no missing data. Potato genotypes are labeled according to the SNP c2_56418 genotype (AAAA/AACC) and stability based on coefficient of variation (CV). Genotypes with an among-experiment CV for sAUDPC of $<0.5$ were considered stable. 
In the specific virulence profiles of the isolates from the two locations, as determined by the differential set, avirulences corresponding to R5 and R9 were frequently present, sometimes together and sometimes separately. Previous reports document a similar trend, that the resistance of differentials R5, R8, and R9 is frequently not overcome $(1,35)$. Our data indicated that pathogen population dynamics are likely driving resistance variability among experiments but it was not clear that these interactions involve any of the $S$. demissum $R$ genes. Furthermore, the instability in resistance reactions was also not associated with reactions of the $S$. demissum differentials. Thus, use of this differential set was not informative for explaining pathogen dynamics relevant to the potato genotypes we assessed. Rather than using the $S$. demissum $R$ gene differential set, a more thorough analysis of the effector combinations and their expression in the isolates present in the study locations could have been more helpful. For the known effectors that act as Avr determinants, this can be readily done $(5,25)$ but, when the $R$ gene- $A v r$ gene pair is unknown, the analysis is not that straightforward.

The low CV of the absolute AUDPC values of Yungay enabled the use of this cultivar to calculate the relative $\mathrm{CV}$ using the sAUDPC as a measure of stability. The stability based on relative $\mathrm{CV}$ is consistent with the location plotting on the PCA bi-plot and with marker analyses. However, it is also important to take into account that stability does not necessarily equate to durability of resistance. We have measured stability of performance in small plots but this is not necessarily a guarantee of durability of resistance should a genotype be planted over much larger areas. Thus, for the genotypes presented here, the durability of resistance remains to be tested.

Based on the pedigree, the resistance linked to the marker c2_56418 could be attributed to the frequent occurrence of the founder clone 575049 (CEW-69-1), which is known as 'Mont- serrat' from Mexico (4). This variety was reported to have stable resistance in Mexico over 5 years and also appears to contain major $R$ genes of an unknown kind (4). However, according to our results, the resistance linked to this marker was not stable because the genotypes with the $\mathrm{C}$ allele in this locus remain resistant only in some of the experiments.

The marker c2_56418 locates toward the end of the long arm of chromosome 9, where we previously mapped a large-effect quantitative trait locus for late blight resistance originating from one of the resistant tetraploid genotypes of the B3 population (22). The physical location of the marker c2_56418 is not in the Rpi-vntl locus but closer to the end of the chromosome. The LD decays relatively rapidly in this part of the chromosome, suggesting that a larger collection of SNPs would be beneficial for better coverage. According to the Potato Genome Sequencing Consortium genome data and Infinium 8303 Potato Array data, the region with multiple copies of Rpi-vntl-like genes locates north of marker c1_14205. The Rpi-vnt1 region contains several SNP markers, of which none were informative in our population, perhaps indicating that the Rpi-vnt1-like genes in the B3 gene pool are more divergent from the Rpi-vntl-like genes found in $S$. venturii and $S$. phureja. Assuming that the increased resistance based on the average sAUDPC in Oxapampa is due to a single locus, the gene located between markers c1_10590 and c1_14205 could be a novel $R$ gene. Recently, the R8 gene from MaR8 was mapped in this genomic region by field inoculation with the $P$. infestans isolate IPO-C (race 1, 2, 3, 4, 5, 6, 7, 10, 11) (19). We showed that, in one of the experiments (Comas2005), the differential set genotype R8 was not infected and, thus, the resistance seen in some of the B3 genotypes that year could be attributed to $\mathrm{R} 8$.

The reference potato genome sequence contains $>400$ nucleotide-binding site leucine-rich repeat-type $R$ genes, and some of

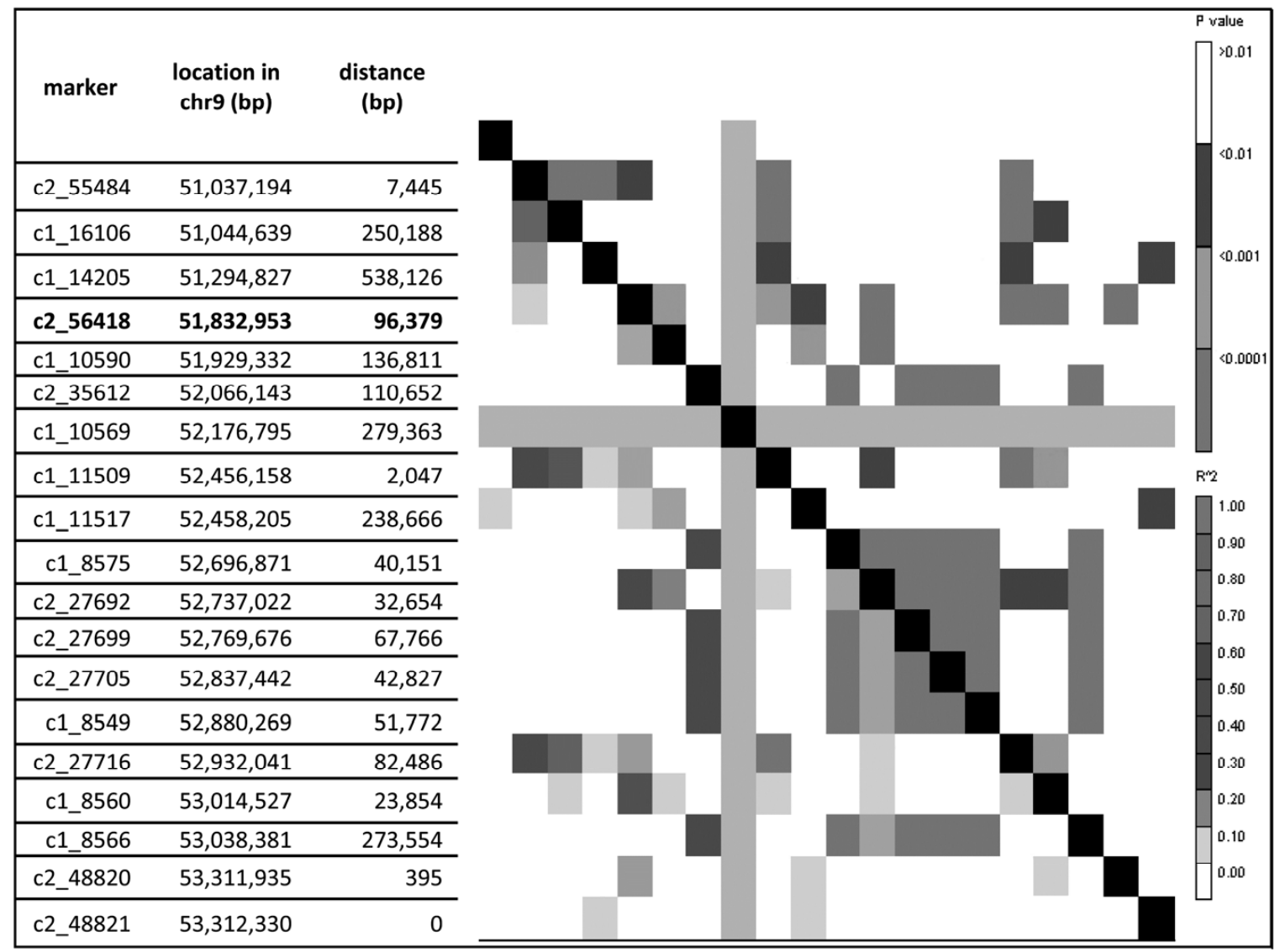

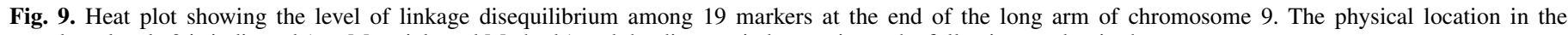
pseudomolecule 9 is indicated (see Materials and Methods) and the distance in base pairs to the following marker is shown. 
those are homologs of the LB resistance genes originally found in distinct Solanum spp. (20). The B3 potato genotypes from CIP are also likely to contain multiple $R$ genes, which may become functional depending on the effector composition of the pathogen population.

The central problem for measuring resistance in breeding populations is that a perfect system for screening using $P$. infestans isolates does not exist. The $P$. infestans genome contains hundreds of effector genes that may have a function in virulence (13). Most of the $P$. infestans isolates described all over the world do show complex virulence patterns in terms of the $11 \mathrm{~S}$. demissum $R$ genes and no doubt also contain more effectors than are associated with the $S$. demissum $-R$ gene interactions. Therefore, we can expect to encounter a mixture of virulence capacities in every isolate and a mixture of $R$ genes in every potato accession, which makes it hard to identify unique gene-for-gene interactions. To further complicate the resistance screening, the phenotype conferred by a major resistance gene is not always clear in detached leaf assays or other controlled inoculations. Recently, it was shown that 'Sarpo Mira' contains four $R$ genes that confer qualitative resistance and an $R$ gene that confers partial resistance, and the resistance phenotype is only detected in field conditions (29). Effectoromics is a promising new tool for $R$ gene identification (37) that could facilitate the monitoring of gene-for-gene phenotypes.

\section{ACKNOWLEDGMENTS}

We thank E. Salas for help in obtaining the historical data and the initial analysis steps, Michigan State University for providing fast and good-quality genotyping service, and the CGIAR CRP RTB for providing funding.

\section{LITERATURE CITED}

1. Andrivon, D. 1994. Race structure and dynamics in populations of Phytophthora infestans. Can. J. Bot. 72:1681-1687.

2. Andrivon, D., Pellé, R., and Ellissèche, D. 2006. Assessing resistance types and levels to epidemic diseases from the analysis of disease progress curves: principles and application to potato late blight. Am. J. Potato Res. 83:455-461.

3. Bradbury, P. J., Zhang, Z., Kroon, D. E., Casstevens, T. M., Ramdoss, Y., and Buckler, E. S. 2007. TASSEL: software for association mapping of complex traits in diverse samples. Bioinformatics 23:2633-2635.

4. Cadena-Hinojosa, M. A., Diaz-Valasis, M., Guzman-Plazola, R. A., Fernandez-Pavia, S., and Grünwald, N. J. 2007. Late blight resistance of five Mexican potato cultivars in the eastern Sierra of the state of Mexico. Am. J. Potato Res. 84:385-392.

5. Clément, J. A. J., Baldwin, T. K., Magalon, H., Glais, I., Gracianne, C., Andrivon, D., and Jacquot, E. 2013. Specific detection and quantification of virulent/avirulent Phytophthora infestans isolates using a real-time PCR assay that targets polymorphisms of the Avr3a gene. Lett. Appl. Microbiol. 56:322-332.

6. Earl, D. A., and vonHoldt, B. M. 2011. STRUCTURE HARVESTER: a website and program for visualizing STRUCTURE output and implementing the Evanno method. Conserv. Genet. Resour. 4:359-361.

7. Felcher, K. J., Coombs, J. J., Massa, A. N., Hansey, C. N., Hamilton, J. P., Veilleux, R. E., Buell, C. R., and Douches, D. S. 2012. Integration of two diploid potato linkage maps with the potato genome sequence. PLoS One 7:e36347.

8. Flor, H. H. 1971. Current status of the gene-for-gene concept. Annu. Rev. Phytopathol. 9:275-296.

9. Forbes, G. A. 2012. Using host resistance to manage potato late blight with particular reference to developing countries. Potato Res. 55:205-216.

10. Forbes, G. A., Chacón, M. G., Kirk, H. G., Huarte, M. A., Van Damme, M., Distel, S., Mackay, G. R., Stewart, H. E., Lowe, R., Duncan, J. M., Mayton, H. S., Fry, W. E., Andrivon, D., Ellissèche, D., Pellé, R., Platt, H. W., MacKenzie, G., Tarn, T. R., Colon, L. T., et al. 2005. Stability of resistance to Phytophthora infestans in potato: an international evaluation. Plant Pathol. 54:364-372.

11. Garry, G., Forbes, G. A., Salas, A., Santa Cruz, M., Perez, W. G., and Nelson, R. J. 2005. Genetic diversity and host differentiation among isolates of Phytophthora infestans from cultivated potato and wild solanaceous hosts in Peru. Plant Pathol. 54:740-748.
12. Garry, G., Salas, A., Forbes, G. A., Perez, W., Cruz, M. S., and Nelson, R. J. 2005. Host specialization not detected among isolates of the ec-1 lineage of Phytophthora infestans attacking wild and cultivated potatoes in Peru. Eur. J. Plant Pathol. 113:71-81.

13. Haas, B. J., Kamoun, S., Zody, M. C., Jiang, R. H. Y., Handsaker, R. E., Cano, L. M., Grabherr, M., Kodira, C. D., Raffaele, S., Torto-Alalibo, T., Bozkurt, T. O., Ah-Fong, A. M. V., Alvarado, L., Anderson, V. L., Armstrong, M. R., Avrova, A., Baxter, L., Beynon, J., Boevink, P. C., Bollmann, S. R., Bos, J. I. B., Bulone, V., Cai, G., Cakir, C., Carrington, J. C., Chawner, M., Conti, L., Costanzo, S., Ewan, R., Fahlgren, N., Fischbach, M. A., Fugelstad, J., Gilroy, E. M., Gnerre, S., Green, P. J., Grenville-Briggs, L. J., Griffith, J., Grünwald, N. J., Horn, K., Horner, N. R., Hu, C.-H., Huitema, E., Jeong, D.-H., Jones, A. M. E., Jones, J. D. G., Jones, R. W., Karlsson, E. K., Kunjeti, S. G., Lamour, K., Liu, Z., Ma, L., MacLean, D., Chibucos, M. C., McDonald, H., McWalters, J., Meijer, H. J. G., Morgan, W., Morris, P. J., Munro, C. A., O'Neill, K., OspinaGiraldo, M., Pinzón, A., Pritchard, L., Ramsahoye, B., Ren, Q., Restrepo, S., Roy, S., Sadanandom, A., Savidor, A., Schornack, S., Schwartz, D. C., Schumann, U. D., Schwessinger, B., Seyer, L., Sharpe, T., Silvar, C., Song, J., Studholme, D. J., Sykes, S., Thines, M., van de Vondervoort, P. J. I., Phuntumart, V., Wawra, S., Weide, R., Win, J., Young, C., Zhou, S., Fry, W., Meyers, B. C., van West, P., Ristaino, J., Govers, F., Birch, P. R. J., Whisson, S. C., Judelson, H. S., and Nusbaum, C. 2009. Genome sequence and analysis of the Irish potato famine pathogen Phytophthora infestans. Nature 461:393-398.

14. Hackett, C. A., McLean, K., and Bryan, G. J. 2013. Linkage analysis and QTL mapping using SNP dosage data in a tetraploid potato mapping population. PLoS One 8:e63939.

15. Hardy, O. J., and Vekemans, X. 2002. spagedi: A versatile computer program to analyse spatial genetic structure at the individual or population levels. Mol. Ecol. Notes 2:618-620.

16. Haverkort, A. J., Struik, P. C., Visser, R. G. F., and Jacobsen, E. 2009. Applied biotechnology to combat late blight in potato caused by Phytophthora infestans. Spec. Issue BIOEXPLOIT Exploit. Nat. Plant Divers. Pestic.-Free Prod. Food 52:249-264.

17. Haynes, K. G., Lambert, D. H., Christ, B. J., Weingartner, D. P., Douches, D. S., Backlund, J. E., Secor, G., Fry, W., and Stevenson, W. 1998. Phenotypic stability of resistance to late blight in potato clones evaluated at eight sites in the United States. Am. J. Potato Res. 75:211-217.

18. Husson, F., Josse, J., and Le, S. 2008. FactoMineR: an R package for multivariate analysis. J. Stat. Softw. 25:1-18.

19. Jo, K.-R., Arens, M., Kim, T.-Y., Jongsma, M. A., Visser, R. G. F., Jacobsen, E., and Vossen, J. H. 2011. Mapping of the S. demissum late blight resistance gene R8 to a new locus on chromosome IX. Theor. Appl. Genet. 123:1331-1340.

20. Jupe, F., Pritchard, L., Etherington, G. J., MacKenzie, K., Cock, P. J., Wright, F., Sharma, S. K., Bolser, D., Bryan, G. J., Jones, J. D., and Hein, I. 2012. Identification and localisation of the NB-LRR gene family within the potato genome. BMC Genomics 13:75.

21. Landeo, J. A., Gastelo, M., Pinedo, H., and Flores, F. 1995. Breeding for horizontal resistance to late blight in potato free of $R$ genes. Pages 268274 in: Phytophthora infestans 150. E. J. Dowley, E. Bannon, L. R. Cooke, T. Keane, and E. O'Sullivan, eds. Boole Press Ltd., Dublin.

22. Li, J., Lindqvist-Kreuze, H., Tian, Z., Liu, J., Song, B., Landeo, J., Portal, L., Gastelo, M., Frisancho, J., Sanchez, L., Meijer, D., Xie, C., and Bonierbale, M. 2012. Conditional QTL underlying resistance to late blight in a diploid potato population. Theor. Appl. Genet. 124:1339-1350.

23. Li, Y., van der Lee, T., Zhu, J. H., Jin, G. H., Lan, C. Z., Zhu, S. X., Zhang, R. F., Liu, B. W., Zhao, Z. J., Kessel, G., Huang, S. W., and Jacobsen, E. 2012. Population structure of Phytophthora infestans in China-geographic clusters and presence of the EU genotype Blue_13. Plant Pathol. 62:932-942.

24. Mulema, J. M. K., Olanya, O. M., Adipala, E., and Wagoire, W. 2004. Stability of late blight resistance in population B potato clones. Potato Res. 47:11-24.

25. Pel, M. A., Foster, S. J., Park, T.-H., Rietman, H., van Arkel, G., Jones, J. D., Van Eck, H. J., Jacobsen, E., Visser, R. G., and Van der Vossen, E. A. 2009. Mapping and cloning of late blight resistance genes from Solanum venturii using an interspecific candidate gene approach. Mol. PlantMicrobe Interact. 22:601-615.

26. Perez, W. G., Gamboa, J. S., Falcon, Y. V., Coca, M., Raymundo, R. M., and Nelson, R. J. 2001. Genetic structure of Peruvian populations of Phytophthora infestans. Phytopathology 91:956-965.

27. Pritchard, J. K., Stephens, M., and Donnelly, P. 2000. Inference of population structure using multilocus genotype data. Genetics 155:945-959.

28. Pule, B. B., Meitz, J. C., Thompson, A. H., Linde, C. C., Fry, W. E., Langenhoven, S. D., Meyers, K. L., Kandolo, D. S., van Rij, N. C., and McLeod, A. 2013. Phytophthora infestans populations in central, eastern and southern African countries consist of two major clonal lineages. Plant Pathol. 62:154-165. 
29. Rietman, H., Bijsterbosch, G., Cano, L. M., Lee, H.-R., Vossen, J. H., Jacobsen, E., Visser, R. G. F., Kamoun, S., and Vleeshouwers, V. G. A. A. 2012. Qualitative and quantitative late blight resistance in the potato cultivar Sarpo Mira is determined by the perception of five distinct RXLR effectors. Mol. Plant-Microbe Interact. 25:910-919.

30. Rodewald, J., and Trognitz, B. 2013. Solanum resistance genes against Phytophthora infestans and their corresponding avirulence genes. Mol. Plant Pathol. 14:740-757.

31. Sharma, B. P., Forbes, G. A., Manandhar, H. K., Shrestha, S. M., and Thapa, R. B. 2013. Determination of resistance to Phytophthora infestans on potato plants in field, laboratory and greenhouse conditions. J. Agric. Sci. Online publication. http://www.ccsenet.org/journal/index.php/jas/ article/view/24430

32. Spielman, L. J., Drenth, A., Davidse, L. C., Sujkowski, L. J., Gu, W., Tooley, P. W., and Fry, W. E. 1991. A second world-wide migration and population displacement of Phytophthora infestans? Plant Pathol. 40:422430.

33. St. Clair, D. A. 2010. Quantitative disease resistance and quantitative resistance loci in breeding. Annu. Rev. Phytopathol. 48:247-268.

34. Stevens, S. S. 1946. On the theory of scales of measurement. Science 103:677-680.

35. Swiezyński, K. M., Domański, L., Zarzycka, H., and Zimnoch-Guzowska, E.
2000. The reaction of potato differentials to Phytophthora infestans isolates collected in nature. Plant Breed. 119:119-126.

36. Umaerus, V., and Umaerus, M. 1994. Inheritance of resistance to late blight. Pages 365-401 in: Potato Genetics. J. E. Bradshaw and G. R. Mackay, eds. CAB International, Wallingford, UK.

37. Vleeshouwers, V. G. A. A., Raffaele, S., Vossen, J. H., Champouret, N., Oliva, R., Segretin, M. E., Rietman, H., Cano, L. M., Lokossou, A., Kessel, G., Pel, M. A., and Kamoun, S. 2011. Understanding and exploiting late blight resistance in the age of effectors. Annu. Rev. Phytopathol. 49:507-531.

38. Voorrips, R. E., Gort, G., and Vosman, B. 2011. Genotype calling in tetraploid species from bi-allelic marker data using mixture models. BMC Bioinf. 12:172.

39. Wulff, E. G., Pérez, W., Nelson, R. J., Bonierbale, M., Landeo, J. A., and Forbes, G. A. 2007. Identification of stable resistance to Phytophthora infestans in potato genotypes evaluated in field experiments in Peru. Exp. Agric. 43:353-363.

40. Xu, X., Pan, S., Cheng, S., Zhang, B., Mu, D., Ni, P., Zhang, G., Yang, S., Li, R., and Wang, J. 2011. Genome sequence and analysis of the tuber crop potato. Nature 475:189.

41. Yuen, J. E., and Forbes, G. A. 2009. Estimating the level of susceptibility to Phytophthora infestans in potato genotypes. Phytopathology 99:782-786. 